\title{
Enhanced Power Sharing Transient with Droop Controllers for Multi-Three-Phase Synchronous Electrical Machines
}

\author{
A. Galassini*, Membership, A. Costabeber*, Membership, M. Degano*, Membership, C. Gerada*†, \\ Membership, A. Tessarolo, Membership, R. Menis, Membership
}

\begin{abstract}
This manuscript presents a droop-based distributed control strategy for multi-three-phase machines that provides augmented controllability during power sharing transients. The proposed strategy is able to mitigate the mutual interactions among different sets of windings without controlling any sub-space variable, also offering a modular and redundant design. On the contrary, in a centralised configuration sub-spaces would be controlled using the Vector Space Decomposition, but fault tolerance and reliability levels required by the stricter regulations and policies expected in future transportation systems would not be satisfied. The proposed method is analytically compared against the state-of-the-art power sharing technique and equivalent models and control design procedures have been derived and considered in the comparison. Uncontrolled power sharing transients and their effects on mutual couplings among isolated sets of windings have been compared against the proposed regulated ones. Experimental results on a $22 \mathrm{~kW}$ nine-phase multi-three-phase synchronous machine rig validate the design procedures showing good agreement with the expected performances.
\end{abstract}

Index Terms-Distributed Power Generation, Motor Drives, Rotating Machines, Variable Speed Drives.

\section{INTRODUCTION}

$\mathbf{M}$ ULTI-PHASE electrical machines are gaining popularity thanks to the on-going electrical propulsion revolution and to the increase of high-power generation demand [1]. They present improved power density, reducing the current per-phase without reaching the power electronics voltage limit [2]-[4]. Power systems for future aerospace and safety critical applications will require higher fault tolerance and reliability levels [5]. Multi-Three-Phase Machines (MTPM) are obtained by grouping multi-phase machine phases into three-phase subwindings sets. Based on how current is controlled within every

Manuscript received Month $\mathrm{xx}, 2 \mathrm{xxx}$; revised Month $\mathrm{xx}, \mathrm{xxxx}$; accepted Month $x, x x x x$. This work was supported in part by the $x x x$ Department of xxx under Grant (sponsor and financial support acknowledgment goes here).

(Authors' names and affiliation) First A. Author1 and Second B. Author2 are with the xxx Department, University of xxx, City, State C.P. Country, on leave from the National Institute for xxx, City, Country (email: author@domain.com).

Third C. Author3 is with the National Institute of xxx, City, State C.P. Country (corresponding author to provide phone: $x x x-x x x-x x x x$; fax: $x x x-$ xxx-xxxx; e-mail: author@ domain.gov).

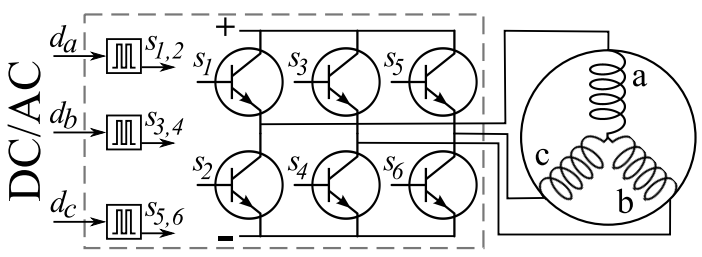

Fig. 1. DC/AC 2-Level 3-Phase Voltage Source Inverter (2L-3P-VSI) and one three phase set of windings $(\mathrm{a}, \mathrm{b}, \mathrm{c}) . d_{a}, d_{b}, d_{c}$ are the duty cycles.

set of windings, better performances or increased redundancy can be achieved. Power-train re-design by adopting MTPM leads to modularity by extending redundancy from the power electronics to the control unit level. The repetition of independent modules made by one three-phase set of windings, one DC/AC two-levels three-phase voltage source inverter (Fig. 1), and one control unit will enable compliance with the forthcoming regulations in aerospace applications.

In this manuscript, a power sharing transient regulator for MTPM controlled by multiple independent modules is proposed, designed and validated experimentally. The sharing transient controllability improves the Total Harmonic Distortion (THD) decreasing mechanical stress, vibrations and Electro-Magnetic-Interferences (EMI) during the power sharing operation.

The paper is organised as follows. In Sec. II, the two typical control configurations for MTPM are introduced and discussed. The conventional power sharing regulator and the proposed one are introduced in Secs. III and IV, respectively. While showing their simplified equivalent models, they are compared in Sec. V. Before deriving the current sharing dynamics and its time constant in Sec. VII, control design procedures are given and compared in Sec. VI. Following two case studies in Sec. VIII, both the regulator designs are validated by means of experimental results in Sec. IX. Conclusions are drawn in Sec. X.

\section{Multi Drive Control Strategies}

In Figs. 2 and 3, two different control configurations for MTPMs are shown. Every DC/AC converter output $\left(v_{a, b, c}\right)$ is connected to one three-phase set of windings, $d_{a, b, c}$ are the duty cycles, $i_{a, b, c}^{\wedge}$ are the measured phase currents, and $\theta$ is the rotor position fed back from the speed sensor to the 


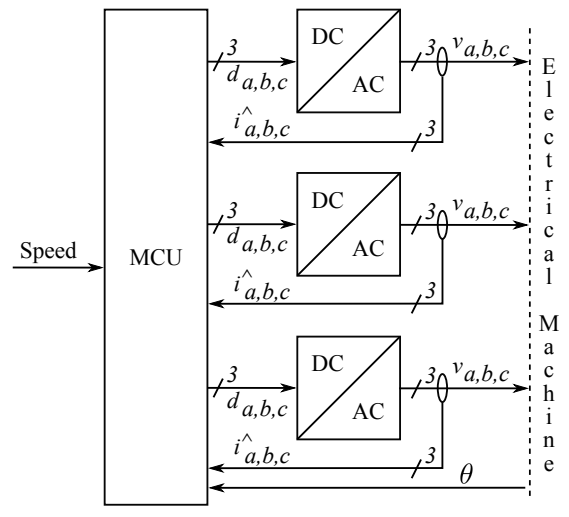

Fig. 2. Centralised control architecture.

Micro Controller Units (MCUs). Thanks to the Vector Space Decomposition (VSD), better current dynamics controlling all the orthonormal sub-spaces can be achieved by the configuration in Fig. 2, in which all the measured currents are fed back into a single MCU. On the other hand, full redundancy is sacrificed in the name of lower Total Harmonic Distortion (THD) [6], in-fact, in case of faulty MCU, the entire system is compromised. Referring to Fig. 3 showing the Common Speed Reference (CSR) configuration, having one MCU per $\mathrm{DC} / \mathrm{AC}$ converter, gives a redundant system. Its main downside is from the controllability point of view. In-fact, sub-spaces can not be controlled because only the three local measured currents are fed back into every single MCU. Considering the higher reliability and fault tolerance levels required by future aerospace systems, the CSR configuration is deemed to be the most suitable one in this paper, since modularity and redundancy are inherently achieved.

\section{Power Sharing - StATE-OF-THE-ART}

Among the features enabled by multi-three-phase systems, like for example augmented reliability and fault tolerance at system level, one of the most interesting is power sharing. In Fig. 4, the CSR simplified control schematic with power sharing capabilities relative to Fig. 3 is shown. Each branch represents the $q$-axis within the rotating reference frame, $\omega^{*}$ is the speed set-point, and $\omega^{\wedge}$ is the measured speed. The continuous transfer functions $\omega_{c} /\left(s+\omega_{c}\right)$ represent the closed $q$-current loops with cut-off frequency $\omega_{c}$ and phase margin $\varphi_{c}$, whilst $\left(s K_{p S}+K_{i S}\right) / s$ blocks are the speed ProportionalIntegral (PI) controller transfer functions. Defining the number of modules $N$, the torque produced by the $j$-th module $T_{j}$ (with $j=1 . . N$ ), the $j$-th $q$-current $i_{q j}$, and the machine constant $K_{t}=T_{j} / i_{q j}$, the total amount of torque $T_{A}$ produced by all the modules is set varying the $q$-current set-points $i_{q j}^{*}$. Introducing a sharing coefficient $W_{j}$, the $j$-th $q$-current setpoint, and therefore $T_{j}$, can be set. The sharing coefficients $W_{j}$ define the new current set-points $i_{q j}^{*^{\prime}}$ described by the following equation: $i_{q j}^{*^{\prime}}=i_{q j}^{*} W_{j}$. In nominal conditions, power is equally split (ES) and loop gains are assumed to be equal to one, $W_{1,2,3}^{(E S)}=1$. Depending on the particular application, unbalanced sharing (US) can be obtained varying the sharing coefficients.

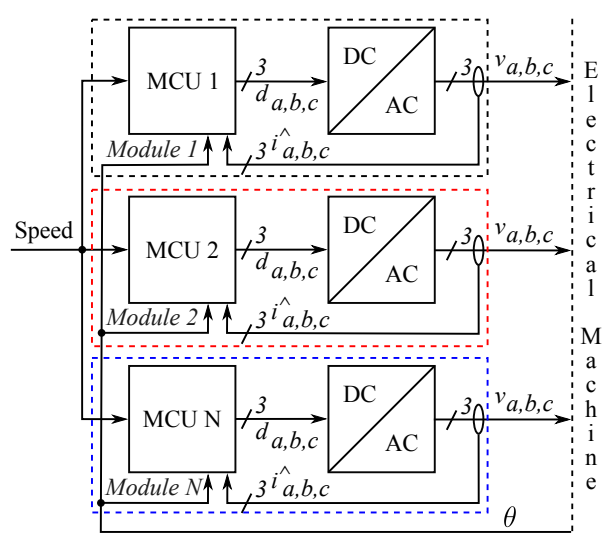

Fig. 3. Common Speed Reference (CSR). Fully modular and redundant.

In order to track the speed set-point $\omega^{*}$, the total power $P_{T O T}$ produced by all the modules must be kept constant. The total power is given by the sum of all the torques produced by each module multiplied by the shaft speed, $P_{T O T}=\sum_{j}^{N} T_{j} \omega$. Defining the global sharing coefficient $W_{T}=\sum_{j}^{N} W_{j}$, the power $P_{j}$ in $p$.u. produced by the $j$-th module is described by the following equation:

$$
P_{j}=\frac{I_{q, j}}{\sum_{j}^{N} I_{q, j}}=\frac{W_{j}}{W_{T}}
$$

where $I_{q, j}$ is the nominal current on the $q$-axis of the $j$-th module. Thanks to (1), as long as $W_{T}$ is kept constant, US can be achieved just changing the sharing coefficients. Whenever US is needed, each $W_{j}$ could be updated by a supervisory controller or programmed off-line a priori using the following formula:

$$
W_{j}^{(U S)}=P_{j}^{(U S)} W_{T}
$$

and until the following equation is verified, constant speed loop dynamics is guaranteed.

$$
W_{T}=W_{T}^{(E S)}=W_{T}^{(U S)}=\sum_{j}^{N} W_{j}=N
$$

\section{ENHANCED POWER SHARING CONTROLLER}

In this work, the droop controller is adopted for controlling the power sharing transient on a MTPM, and it is compared against the power sharing transient obtained by stepping the

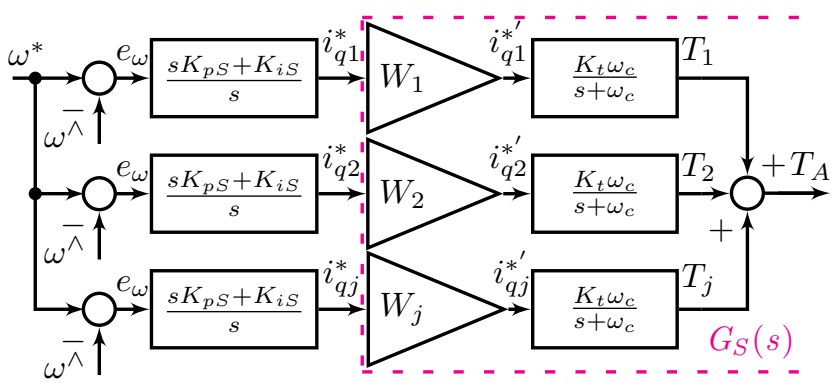

Fig. 4. CSR simplified control schematic with power sharing capabilities. 


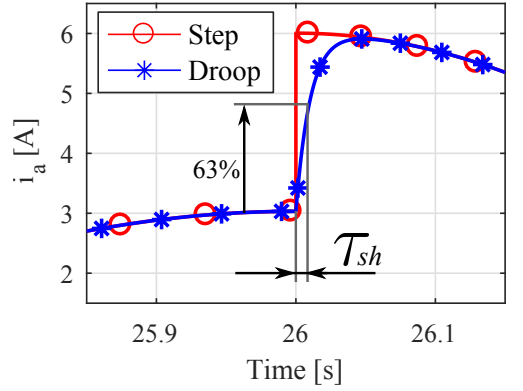

Fig. 5. Example of currents on phase $a$ during power sharing transients (droop vs. step).

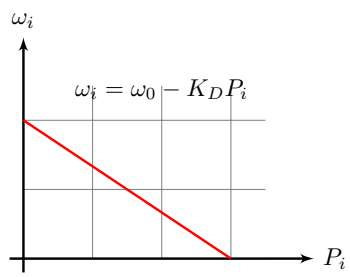

(a) Droop plane in power systems.

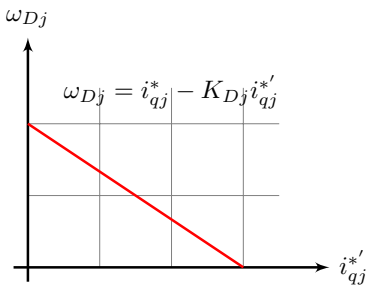

(b) Droop plane in motor control.
Fig. 6. Droop planes comparison.

power sharing coefficients as previously discussed in Sec. III. As a qualitative example to anticipate the power sharing transients that will be shown later, Fig. 5 shows the same power sharing transient from $3[A]$ to $6[A]$ processed by the power sharing coefficient and by the droop controller. It can be observed that the droop controller enables the power sharing transient to be controlled by setting the time constant $\tau_{s h}$, thus helping reducing vibrations and EMI.

Droop control is a very well known technique adopted in power systems allowing the power demanded by the grid to be shared among different generators [7]. The droop characteristic is a linear function with negative coefficient $K_{D}$, called droop coefficient, on the Frequency-Active Power plane shown in Fig. 6a governed by [8]:

$$
\omega_{i}=\omega_{0}-K_{D} P_{i}
$$

where $\omega_{i}$ and $\omega_{0}$ are the angular frequency of the output voltage and the nominal one, respectively, and $P_{i}$ is the output active power (Fig. 6a). The greater the frequency, the less the power produced by the generation plant. Generated power can therefore be partitioned power among generation plants as a function of the droop gains [9].

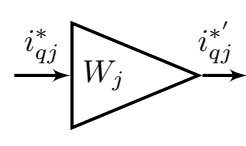

(a) Sharing coefficient

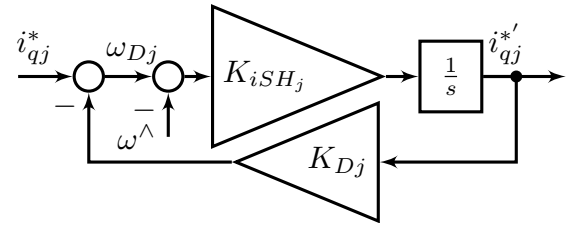

(b) Droop controller $\left(G_{D j}\right)$
Fig. 7. Sharing regulator control diagrams comparison.
In multi-three-phase systems, the droop characteristic of the $j$-th module (Fig. 6b) is defined by the current reference $i_{q j}^{*}$, set by the speed regulator, and by the new current set-point $i_{q j}^{*^{\prime}}$ according to:

$$
\omega_{D j}=i_{q j}^{*}-K_{D j} i_{q j}^{*^{\prime}}
$$

where $\omega_{D j}$ is an internal drooped set-point.

The control diagram relative to (5) is shown and compared against the sharing coefficient one in Fig. 7. Considering the control diagram in Fig. $7 \mathrm{~b}$, the $j$-th droop controller inputoutput relation is described by the following transfer function:

$$
G_{D j}(s)=\frac{K_{i S H j}}{s+K_{i S H j} K_{D j}}=\frac{i_{q j}^{*^{\prime}}}{i_{q j}^{*}}
$$

where $K_{i S H j}$ is the integral droop coefficient. For simplicity, considering a system with only two modules, the enhanced Common Speed Reference (eCSR) control diagram is shown in Fig. 8. The mechanical load is common and it is represented by a linear model with inertia $J$ and friction $F$. Like in Fig. 4, every $q$-current control loop is modelled as a lowpass filter with bandwidth $\omega_{c}$ and phase margin $\varphi_{c}$. In the $e$ CSR configuration shown in Fig. 8 there are the following regulators:

1) Proportional-Integral $q$-current controllers

$$
P I_{I q}=K_{p I q}+K_{i I q} / s
$$

within the $q$-current loops modelled by transfer functions $\omega_{c} /\left(s+\omega_{c}\right)$ as discussed in Sec. III;

2) Speed-drooped, or droop or sharing, controllers $G_{D j}$ shown in Fig. $7 \mathrm{~b}$ characterised by bandwidth and phase margin $\omega_{S H}$ and $\varphi_{S H}$, respectively, and described by (6);

3) Proportional-Integral speed, controllers

$$
P I_{S}=\left(s K_{p S}+K_{i S}\right) / s
$$

characterised by bandwidth and phase margin $\omega_{s}$ and $\varphi_{s}$, respectively.

\section{A. Global sharing coefficient}

In order to better understand Sec. VII, it is important to take into consideration the difference between steady state gains of controllers from Fig. 7. For $s \rightarrow 0$, whilst the gain of the conventional controller is the sharing coefficient $W_{j}$ itself, looking at (6) for $s \rightarrow 0$, the gain of the proposed droop controller $G_{D j}$ is $1 / K_{D j}$. The resulting global sharing coefficient for the enhanced power sharing controller is expressed by the following formula:

$$
W_{T}^{\prime}=\sum_{j}^{N}\left(1 / K_{D j}\right)
$$

\section{B. Speed drop}

Considering the control diagram in Fig. 8, but without the outermost speed loops, the final speed of the shaft at steady state can be calculated taking the limit for $s \rightarrow 0$ :

$$
\omega=\omega^{*} \frac{\gamma}{\gamma+1}-\frac{T_{L}}{F+W_{T}^{\prime} K_{t}} \text { with } \gamma=\frac{W_{T}^{\prime} K_{t}}{F}
$$




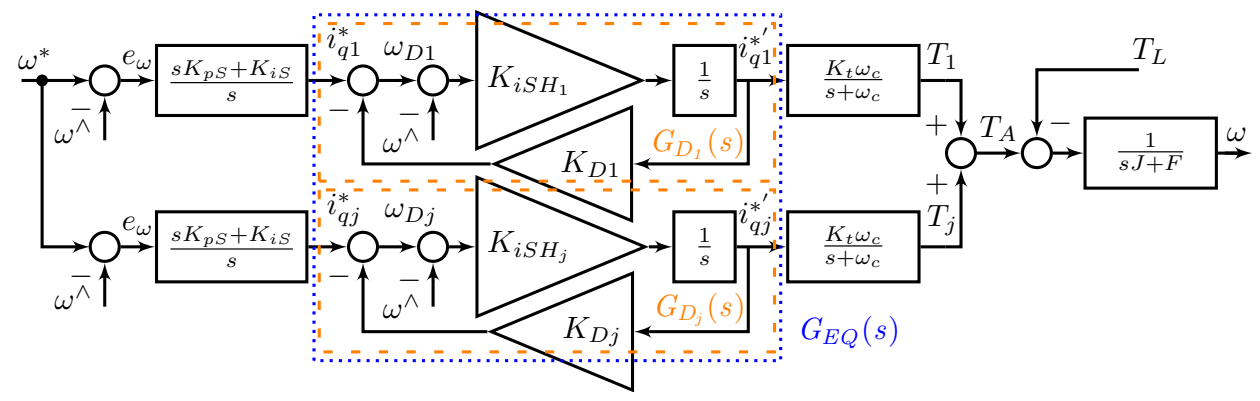

Fig. 8. Common Speed Reference control diagram with enhanced, or droop, controllers (eCSR). For simplicity, only two modules are shown.

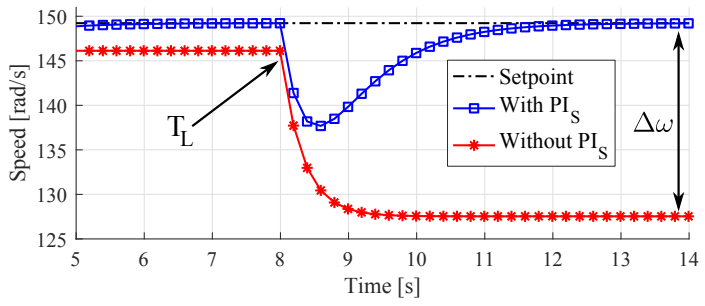

Fig. 9. The grater the torque demand, the greater the speed drop.

Looking at Figs. 6, in power systems the greater the power $P_{i}$ produced by the $i-t h$ generation plant, the less the angular frequency $\omega_{i}$. In motor control, the greater the $q$-current setpoint $i_{q j}^{*}$, the less the internal drooped set-point $\omega_{D j}$. In other words, looking at Fig. 9, the greater the torque demand, the greater the speed drop defined as $\Delta \omega=\omega^{*}-\omega$. For a given maximum torque load, the $\Delta \omega_{M A X}$ would be the maximum speed drop if the outermost speed loops were not in place.

\section{Simplified EquiVALENT Models}

In general, current regulators in MTPMs are designed on harmonic inductance and phase resistance values [10]. By definition, the first $d$ and $q$ harmonic inductances are mapped into the $\alpha-\beta$ plane and they are the inductances seen by the DC/AC converters under ideal and balanced voltage supply hypothesis [11]. The harmonic inductances can be obtained thanks to the VSD, and once they are computed, PI controllers along $d$ and $q$ axes can be designed taking into account actuation and filtering delays. The design of paralleled speed regulators shown in Figs. 4 and 8 is done assuming the power is equally split among all the modules. Looking at Figs. 4 and 8 , the $j$-th speed controller output is the input reference $i_{q j}^{*}$ for the $j$-th sharing controller. In the following sub-sections, reduced equivalent models for designing the speed controllers will be introduced.

\section{A. Common Speed Reference}

Looking at Fig. 4, and assuming the power is equally split among the modules $\left(W_{1,2,3}^{(E S)}=1\right)$, the simplified equivalent model taking into account the $N$ paralleled branches is shown and highlighted within dashed square in Fig. 10. Design of the speed regulators $P I_{S}$ can be done on the following plant:

$$
G_{S}(s)=N \frac{\omega_{c}}{s+\omega_{c}} K_{t} \frac{1}{s J+F}
$$

The same parameters $K_{p S}^{(C S R)}$ and $K_{i S}^{(C S R)}$ computed on plant in (11) can be used in the CSR simplified control schematic shown in Fig. 4.

\section{B. Enhanced Common Speed Reference}

Like in the previous sub-section, speed and droop controller design has to be done on a plant considering the whole system and assuming equal power sharing $\left(K_{D_{1}}^{(E S)}=K_{D_{j}}^{(E S)}\right.$ and $\left.K_{i S H_{1}}^{(E S)}=K_{i S H_{j}}^{(E S)}\right)$. To this purpose, the control diagram in Fig. 8 can be simplified with the collective one shown in Fig. 11. The parallel of the $N$ droop controllers $G_{D j}^{(E S)}$ has been replaced by the equivalent collective droop controller:

$$
G_{E Q}(s)=N G_{D j}^{(E S)}(s)
$$

within the dotted blue square in Fig. 11. The constraint in (12) can be satisfied if and only if $K_{i S H}=K_{i S H j}^{(E S)} N$ and $K_{D}=K_{D j}^{(E S)} / N$, as highlighted by the following equation:

$$
G_{E Q}(s)=\frac{N K_{i S H j}^{(E S)}}{s+K_{i S H j}^{(E S)} N \frac{K_{D j}^{(E S)}}{N}}=N G_{D j}^{(E S)}(s)
$$

From (13), it can be verified that the whole system can be modelled as an equivalent single module with integral sharing gain $K_{i S H}$ and droop gain $K_{D}$ :

$$
K_{i S H}=K_{i S H_{j}}^{(E S)} N \quad K_{D}=\frac{K_{D_{j}}^{(E S)}}{N}
$$

The $K_{D}$ and the $K_{i S H}$ gains in (14) can be defined as the collective droop and the collective integral gain coefficient, respectively. The equivalence in (12) can be further verified by plotting the Bode diagrams in Fig. 12. Defining the following transfer function:

$$
G_{S H_{O L}}(s)=G_{E Q}(s) \frac{\omega_{c}}{s+\omega_{c}} K_{t} \frac{1}{s J+F}
$$

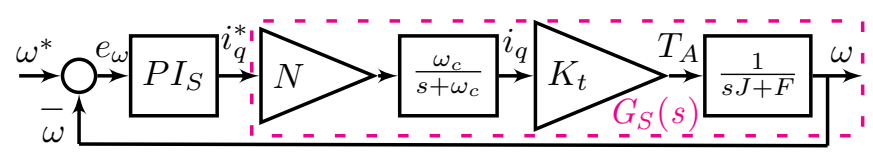

Fig. 10. Simplified equivalent model assuming sharing coefficients $W_{1,2,3}^{(E S)}=1$. For simplicity, the mechanical model is not shown in Fig. 4. 


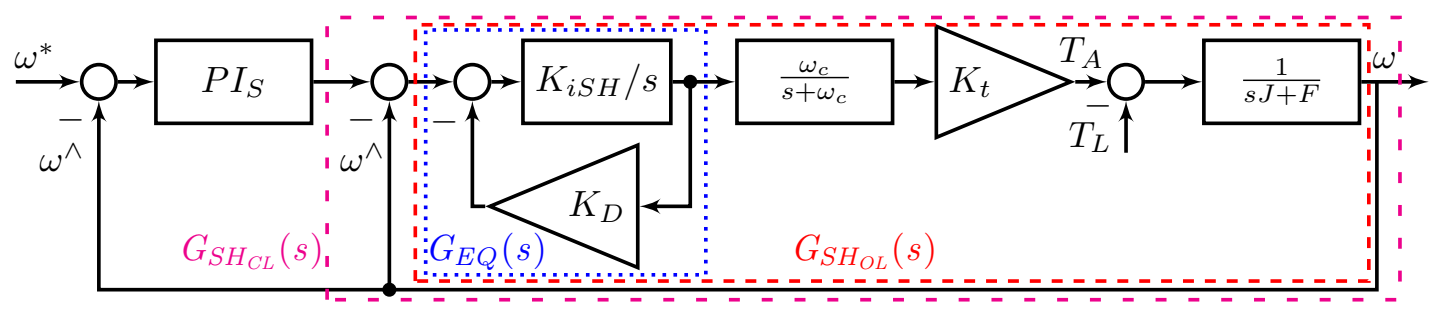

Fig. 11. Equivalent collective control scheme for the eCSR configuration assuming the power is equally split among the $N$ modules.

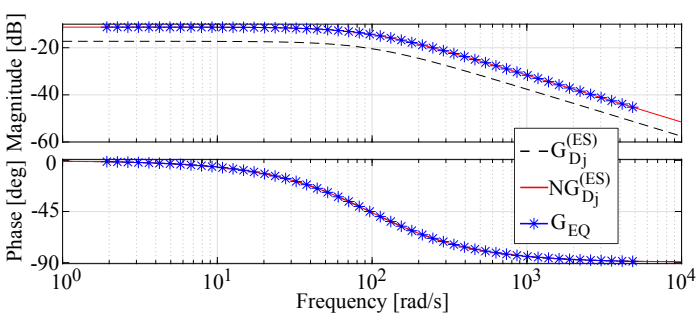

Fig. 12. Bode diagrams of transfer functions in (12).

Therefore, speed regulator design can be done on the plant:

$$
G_{S H_{C L}}(s)=G_{S H_{O L}}(s) /\left(1+G_{S H_{O L}}(s)\right)
$$

Both transfer functions in (15) and (16) are highlighted in Fig. 11 by dashed red square and loosely dashed magenta square, respectively.

\section{Vi. Control Design Procedures}

Considering a system with $N$ modules and with equal power sharing, design procedures for both control diagrams in Figs. 10 and 11 here are discussed and compared. It is important to notice that the $d q$-current loops denoted by low pass filters $\omega_{c} /\left(s+\omega_{c}\right)$ and their design are the same for both control schemes. The plant used for designing the current $P I$ regulators is expressed by Eq. (17). Measurement delay has been modelled by a second order filter with cut-off frequency $\omega_{f c}$, whilst actuation delay has been shaped as a pure delay $e^{-s 1.5 T_{s}}$, where $T_{s}$ is the switching period. $d q$ current regulators are designed imposing bandwidth $\omega_{c}$ and phase margin $\varphi_{c}$ on the following plant:

$$
G_{I \Lambda}(s)=e^{-s 1.5 T_{s}} \frac{1}{s \Lambda_{1}+r_{s}} \frac{\omega_{f c}^{2}}{s^{2}+\sqrt{2} \omega_{f c} s+\omega_{f c}^{2}}
$$

where $r_{s}$ is the phase stator resistance, $\Lambda$ stands for $d$ or $q$, and $\Lambda_{1}$ is the first harmonic inductance along the $d$ or $q$ axis calculated using the VSD.

\section{A. Common Speed Reference}

Once the $d q$-current regulators are designed on plant $G_{I \Lambda}(s)$, speed regulators setting the sharing controller $q$ current references are designed on plant $G_{S}(s)$ in (11) considering the equivalent control scheme in Fig. 10. The same PI gains $K_{p S}^{(C S R)}$ and $K_{i S}^{(C S R)}$ can be used into control schematic in Fig. 4, where $W_{1,2,3}$ were assumed to be equal to one. While keeping constant the global sharing coefficient $W_{T}$ in (3), power sharing can be then achieved by setting different sharing coefficient $W_{j}$ thanks (1) and (2).

\section{B. Enhanced Common Speed Reference}

The introduction of droop controllers in between $q$-current and speed loops allows the power sharing transient to be regulated. The collective droop coefficient $K_{D}$ must be chosen with the following equation:

$$
K_{D}=\Delta \omega_{M A X} / I_{T O T}=\Delta \omega_{M A X} / \sum_{j}^{N} I_{q, j}
$$

where $\triangle \omega_{M A X}$ is the steady state speed error without the outermost speed loops at full load, and $I_{q, j}$ is the nominal current on the $q$-axis of the $j$-th module. Referring to Fig. 11, for a given sharing bandwidth $\omega_{S H}$, sufficiently slower than the current dynamics and faster than the outer speed loop, the collective integral gain $K_{i S H}$ can be calculated imposing the phase margin $\varphi_{S H}$ on (15), leading to the following analytical expression for the collective integral gain:

$$
K_{i S H}=\frac{\omega_{S H}}{\tan \left[-\varphi_{S H}+\pi-\operatorname{atan}\left(\frac{\omega_{S H}}{\omega_{c}}\right)-\operatorname{atan}\left(\frac{\omega_{S H} J}{F}\right)\right]_{(19)} K_{D}}
$$

Under the previous hypothesis of balanced load and provided that $G_{E Q}=N G_{D j}^{(E S)}$, the same response of the designed equivalent collective system when using $N$ modules can be achieved multiplying by $N$ the collective droop gain and dividing by $N$ the collective integral gain like in (14). Once the collective sharing regulator is designed and its relative permodule coefficients $K_{D_{j}}^{(E S)}$ and $K_{i S H_{j}}^{(E S)}$ are computed, speed regulators design can be done considering the plant $G_{S H_{C L}}(s)$ in (16).

\section{DROOP SLOPES AND CURRENT SHARING DYNAMICS}

Droop and speed loop regulators design is done under equal sharing hypothesis. If in the CSR configuration unbalanced sharing is achieved changing $W_{1,2,3}^{(U S)} \neq 1$ considering (3), in the $e$ CSR configuration power sharing is achieved changing $K_{D_{j}}$. However, constant speed loop bandwidth and phase margins are guaranteed if and only if $K_{i S H_{j}}$ are modified accordingly.

Defining $W_{j}^{\prime}=1 / K_{D j}$, the power in $p . u$. produced by the $j$-th module is described by the following equation:

$$
P_{j}=\frac{I_{q, j}}{\sum_{j}^{N} I_{q, j}}=\frac{1 / K_{D j}}{\sum_{j}^{N}\left(1 / K_{D j}\right)}=\frac{W_{j}^{\prime}}{W_{T}^{\prime}}
$$

Comparing (20) against (1), in contrast to the CSR configuration, $P_{j}$ is decreased by setting a bigger droop coefficient $K_{D j}$. 


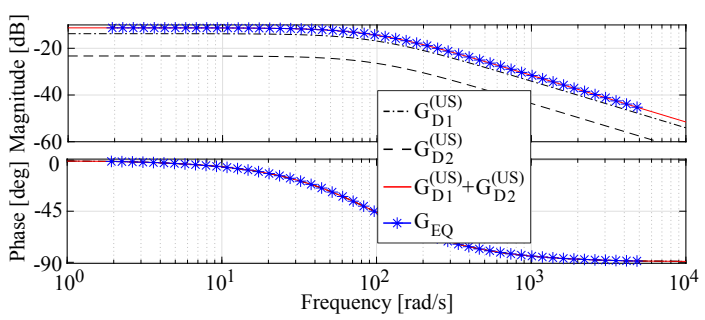

Fig. 13. Updating the integral gains $K_{i S H j}$, constant frequency response with different power ratios is guaranteed.

Vice-versa, higher power is achieved with a smaller droop coefficient. Looking (6), solely updating the droop coefficient would affect the droop controller transfer function frequency response, and therefore the collective one too. Equivalent droop controller constant frequency response is guaranteed if and only if the following condition is verified:

$$
\sum_{j}^{N} G_{D j}^{(U S)}(s)=N G_{D j}^{(E S)}(s)=G_{E Q}(s)
$$

On the assumption that $\sum_{j}^{N} P_{j}=1$, (21) can be satisfied by dividing the individual equal power droop coefficients $K_{D j}^{(E S)}$ by a factor:

$$
\xi_{j}=N P_{j}
$$

and multiplying the individual integral gain $K_{i S H j}^{(E S)}$ by the same factor $\xi_{j}$. Combining (6) and (21):

$$
\frac{N K_{i S H j}^{(E S)}}{s+K_{i S H j}^{(E S)} K_{D j}^{(E S)}}=\sum_{j}^{N} \frac{\overbrace{K_{i S H j}^{(E S)} \xi_{j}}^{K_{i S H j}^{(U S)}}}{s+\underbrace{K_{i S H j}^{(E S)} \xi_{j}}_{K_{i S H j}^{(U S)}} \underbrace{\frac{K_{D j}^{(E S)}}{\xi_{j}}}_{K_{D j}^{(U S)}}}
$$

Equivalences in (21) and (23) are highlighted by their relative Blode plots in Fig. 13. The droop controller power sharing transient is ruled by the time constant $\tau_{s h, j}$ defined by the following equation:

$$
\tau_{s h, j}=\frac{1}{K_{D j} K_{i S H j}}
$$

\section{Control Design - CASE Studies}

In both CSR and $e$ CSR configuration, current regulators have been designed considering the plant $G_{I \Lambda}(s)$ in (17). In the two following case studies, power sharing has been performed setting the following power ratios: $P_{1}=2 / 3$, $P_{2}=1 / 12$, and $P_{3}=1 / 4$.

\section{A. Common Speed Reference}

Assuming $W_{1,2,3}=1$, speed controller for the CSR configuration has been designed on plant $G_{S}(s)$ in (11) leading to $K_{p S}^{(C S R)}$ and $K_{i S}^{(C S R)}$. Provided that $W_{T}=N=3$, power sharing is achieved with (2) leading to the following sharing coefficients: $W_{1}=2, W_{1}=0.25$, and $W_{3}=0.75$, reported in Table I.

\section{B. Enhanced Common Speed Reference}

Speed controller design has to be done after that the equivalent collective sharing controller $G_{E Q}(s)$ in (13) is arranged. Thanks to (18) and (19), the resulting collective droop and integral coefficients can be computed. In order to highlight that the droop controller allows the power sharing time constant to be controlled, $G_{E Q}(s)$ will be designed two times with two different sets of design input parameters $\left(\omega_{S H}\right.$ and $\left.\varphi_{S H}\right)$, labelled fast ${ }^{(f)}$ and slow ${ }^{(s)}$, respectively. Eqs. (18) and (19) are leading to the following collective coefficients: $K_{D}^{(f)}=K_{D}^{(s)}=0.5, K_{i S H}^{(f)}=2000$, and $K_{i S H}^{(s)}=66 . \overline{6}$. Once the equivalent sharing controller is obtained, the speed controller can be designed on the plant $G_{S H_{C L}}(s)$ in (16). Since the collective droop controller has been designed with two different sets of design input parameters $\left(G_{E Q}^{(f)}(s)\right.$ and $G_{E Q}^{(s)}(s)$ ), two distinct sets of speed controller coefficients have been computed $\left(K_{p S}^{(f)}, K_{i S}^{(f)}, K_{p S}^{(s)}, K_{i S}^{(s)}\right)$.

Per-module ES droop coefficients are then obtained by

\begin{tabular}{|c|c|c|c|c|c|c|c|c|c|c|c|}
\hline \multirow[b]{2}{*}{$j$} & \multicolumn{3}{|c|}{$\operatorname{CSR}\left(\tau_{s h, j}^{(C S R)} \rightarrow 0\right)$} & \multicolumn{4}{|c|}{$\begin{array}{l}\text { Fast Sharing }\left(\tau_{s h, j}^{(f)}=1[m s]\right) \\
K_{D}^{(f)}=0.5 \text { and } K_{i S H}^{(f)}=2000\end{array}$} & \multicolumn{4}{|c|}{$\begin{array}{l}\text { Slow Sharing }\left(\tau_{s h, j}^{(s)}=30[m s]\right) \\
K_{D}^{(s)}=0.5 \text { and } K_{i S H}^{(s)}=66 . \overline{6}\end{array}$} \\
\hline & $P_{j}$ & $i_{q j}$ & $W_{j}$ & $P_{j}$ & $i_{q j}$ & $K_{D j}$ & $K_{i S h j}$ & $P_{j}$ & $i_{q j}$ & $K_{D j}$ & $K_{i S h j}$ \\
\hline \multicolumn{12}{|c|}{ Time: $0 s \div 17.5 s$ - Equal Sharing (ES) $-\left(K_{D j}^{(E S)}=K_{D} N\right.$ and $\left.K_{i S H j}^{(E S)}=K_{i S H} / N\right)$} \\
\hline $1,2,3$ & $1 / 3$ & 2 & 1 & $1 / 3$ & 2 & 1.5 & $666 . \overline{6}$ & $1 / 3$ & 2 & 1.5 & $22 . \overline{2}$ \\
\hline$\sum$ & 1 & 6 & $W_{T}=3$ & 1 & 6 & $W_{T}^{\prime}=2$ & 2000 & 1 & 6 & $W_{T}^{\prime}=2$ & $66 . \overline{6}$ \\
\hline \multicolumn{12}{|c|}{ Time: $17.5 s \div 20.5 s$ - Unbalanced Sharing (US) $-\left(K_{D j}^{(U S)}=K_{D j}^{(E S)} / \xi_{j}\right.$ and $\left.K_{i S H j}^{(U S)}=K_{i S H j}^{(E S)} \xi_{j}\right)$} \\
\hline 1 & $2 / 3$ & 4 & 2 & $2 / 3$ & 4 & 0.75 & $1333 . \overline{3}$ & $2 / 3$ & 4 & 0.75 & $44 . \overline{4}$ \\
\hline 2 & $1 / 12$ & 0.5 & 0.25 & $1 / 12$ & 0.5 & 6 & $166 . \overline{6}$ & $1 / 12$ & 0.5 & 6 & $5 . \overline{5}$ \\
\hline 3 & $1 / 4$ & 1.5 & 0.75 & $1 / 4$ & 1.5 & 2 & 500 & $1 / 4$ & 1.5 & 2 & $16 . \overline{6}$ \\
\hline$\sum$ & 1 & 6 & $W_{T}=3$ & 1 & 6 & $W_{T}^{\prime}=2$ & 2000 & 1 & 6 & $W_{T}^{\prime}=2$ & $66 . \overline{6}$ \\
\hline
\end{tabular}
multiplying and dividing the collective gains by $N$ like in

TABLE I

Sharing Controller PARAmeters 


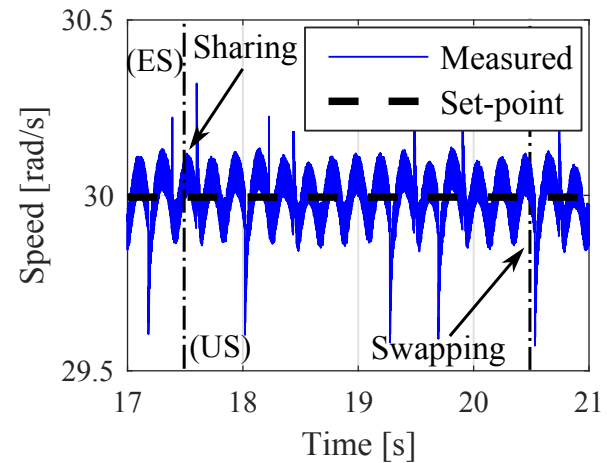

(a) Speed is not affected by load sharing.

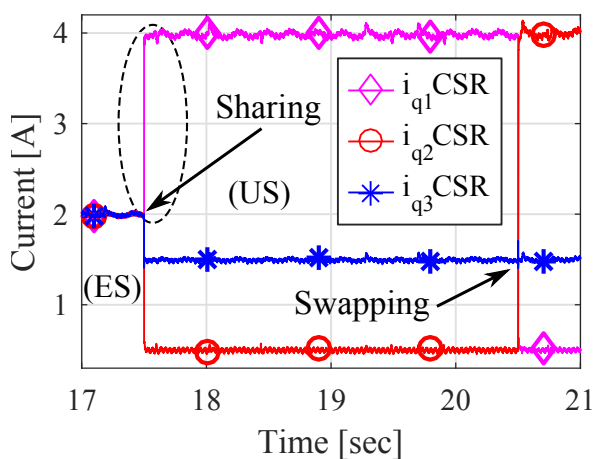

(b) $q$-currents under US conditions from CSR mode.(c) Phase $a$ current transients while swapping $W_{1,2}$.

Fig. 14. CSR measurements. In Fig. 14a, constant speed during sharing and swapping operation is highlighted. Un-controlled $q$-current transients during sharing and swapping operations are shown in Fig. 14b. In Fig. 14c, respective $a$ phase currents during swapping operation are shown.

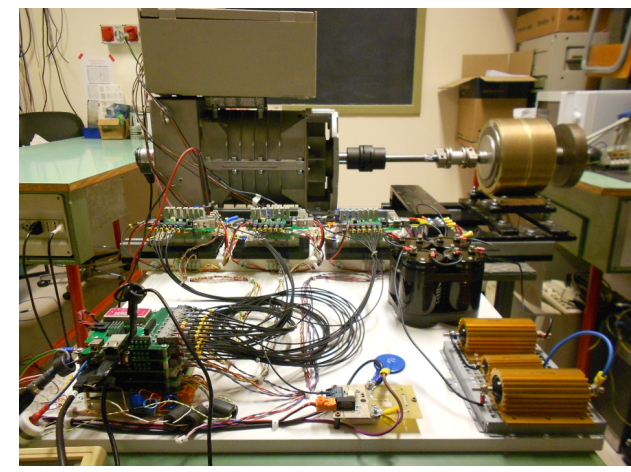

Fig. 15. Multi-three-phase rig with nine phases.

(14), leading to the followings: $K_{D j}^{(E S, f)}=K_{D j}^{(E S, s)}=1.5$, $K_{i S H j}^{(E S, f)}=666 . \overline{6}$, and $K_{i S H j}^{(E S, s)}=22 . \overline{2}$. The relative power sharing time constants can be obtained by (24) leading to $\tau_{s h, j}^{(f)}=1[m s]$ and $\tau_{s h, j}^{(s)}=30[m s]$. Finally, per-module US sharing coefficients have been computed by putting the power ratios into (22), and then multiplying and dividing by $\xi_{j}$ the integral and the droop coefficient, $K_{i S H j}^{(E S)}$ and $K_{D j}^{(E S)}$, respectively, like in (23). All the sharing controller parameters are summarised in Table I.

\section{EXPERIMENTAL VALIDATION}

The droop controller has been validated on the experimental rig in Fig. 15. Every module was independently controlled by a custom control platform. The MTPM with nine phases in Fig. 15 is a two poles synchronous machine.

Switching and sampling frequency were both set to $10[k H z]$, braking torque generated by the hysteresis brake was $55.2[\mathrm{Nm}]$, rotor field DC current was $1.58[\mathrm{~A}]$, and DC link voltage was $350[\mathrm{~V}]$.

The measured output speed while performing power sharing with coefficients from Table I with modules in CSR mode is shown in Fig. 14a.

The $q$ and their respective $a$ phase currents from the three modules are plotted in Figs. 14b and 14c, respectively. Until $17.5[s]$, having set $W_{1,2,3}=1$, power was equally shared among the modules. From $17.5[s]$ and $20.5[s]$, sharing coefficients $W_{1,2,3}$ have been set equal to $2,0.25$, and 0.75 , respectively. At instant 20.5[s] sharing coefficients $W_{1}$ and $W_{2}$ have been swapped. Looking at Fig. 14a, it is possible to verify that the speed is not affected by the power sharing and swapping operations.

In Fig. 16, measured speeds and $q$-currents from the modules configured in $e$ CSR mode are shown. Looking at the measured output speeds during sharing and swapping operations for different time constants $\tau_{s h}$ shown in Fig. 16a,

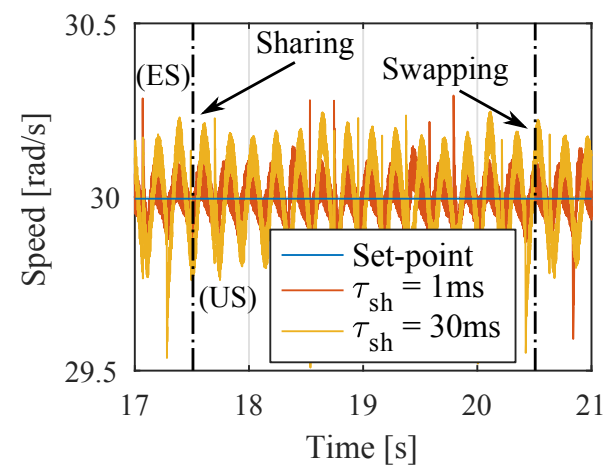

(a) Measured speeds for different time constants.

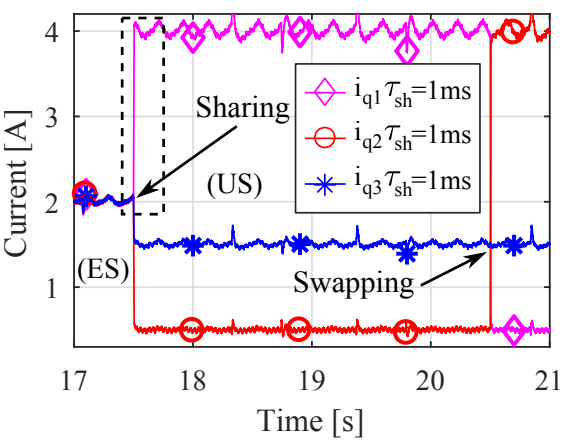

(b) $i_{q}$ currents from $e \mathrm{CSR}$ mode with $\tau_{s h}=1 \mathrm{~ms}$.

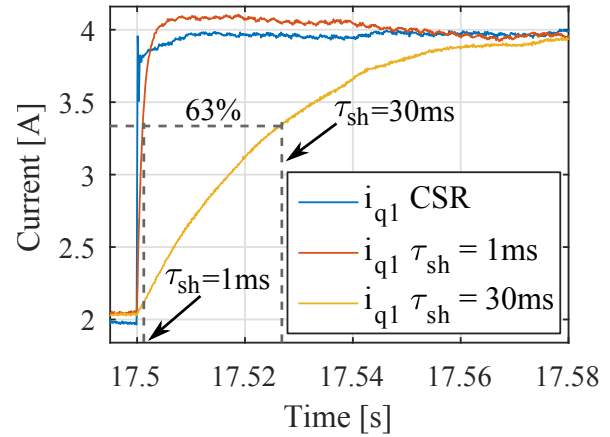

(c) CSR against $e$ CSR for different time constants.

Fig. 16. eCSR measurements. In Fig. 16a, constant speeds during sharing and swapping operation are highlighted. Respective controlled $q$-current transients with $\tau_{s h}=1[\mathrm{~ms}]$ are shown in Fig. 14b. Un-controlled transient $i_{q 1}$ CSR is compared against the controlled ones in Fig. 16c. 
it is possible to verify that the speed is not affected by the droop controllers. The $q$-currents under the same power sharing profile of Fig. 14b but with modules configured in $e$ CSR mode with $\tau_{s h}=1[\mathrm{~ms}]$ are shown in Fig. 16b. In Fig. 16c, the ucurrent transient in blue, labelled $i_{q 1} C S R$ and highlighted by the dashed oval in Fig. 14b, is compared against the controlled current transients for different sharing time constants. The controlled transient plotted in red and labelled $i_{q 1} \tau_{s h}=1 \mathrm{~ms}$, is highlighted by the dashed square in Fig. 16b. The $q$-current steady state values in Figs. 14b and $16 \mathrm{~b}$, together with rise times in Fig. 16c, are validating the control design procedures and the current sharing dynamics discussed in Secs. VI and VII, respectively.

Phase a currents during swapping operations for $\tau_{s h}=$ $1[\mathrm{~ms}]$ and $\tau_{s h}=30[\mathrm{~ms}]$ are zoomed in Figs. 17 and 18 , respectively. In both the plots, currents from the $e \mathrm{CSR}$ (continuous lines) are compared against currents from the CSR (dashed lines) and highlighted by the dashed oval in Fig. 14c. Looking at both Fig. 17 and 18, the $a$ current distortion from the third module, labelled $i_{a 3}(\mathrm{CSR})$, can be noticed. The distortion is caused by the mutual interactions among different sets of windings within the stator. In Fig. 17, the droop controller clearly mitigates the distortion caused by the electro-magnetic coupling. By increasing the sharing time

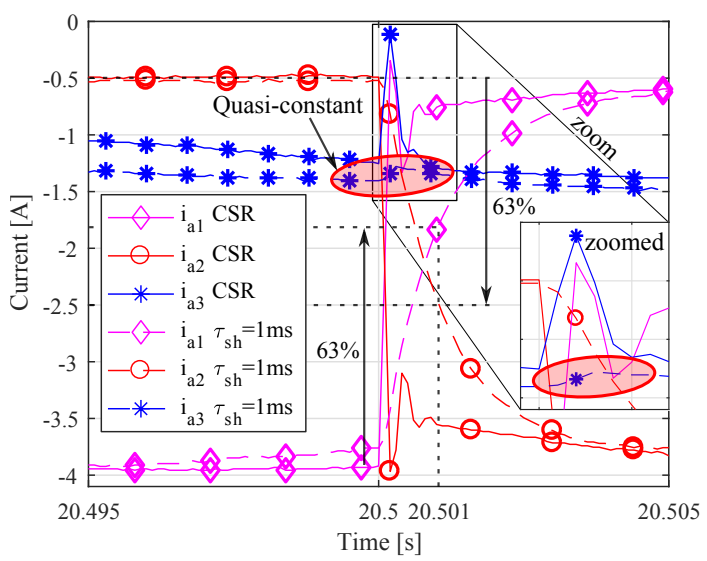

Fig. 17. Common speed reference versus speed-drooped phase currents with $\tau=1 \mathrm{~ms}$ under swapping operation.

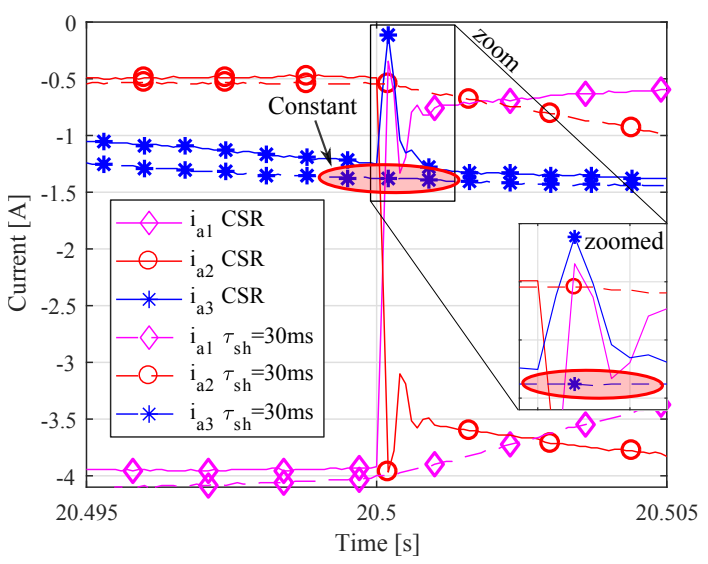

Fig. 18. Common speed reference versus speed-drooped phase currents with $\tau=30 \mathrm{~ms}$ under swapping operation. constant $\tau_{s h}$ to $30[\mathrm{~ms}]$, the third $a$ current from quasi-constant it becomes constant, like highlighted by Fig. 18

\section{Conclusions}

This manuscript is focused on the power sharing transient and its control design for multi-three-phase electrical machines. The proposed enhanced distributed configuration allows the power sharing time constant to be set, and during power sharing transients, current distortion due to the mutual interactions among different sets of windings is mitigated, thus reducing vibrations and electro-magnetic-interferences. Power sharing transient controllability is discussed and successfully verified after introducing the droop regulator for motor control applications. The droop controller and its characteristic was derived from the power system field where is very well known and widely adopted. Transfer functions, simplified equivalent models, Bode diagrams, and design procedures for both the Common Speed Reference and the proposed enhanced Common Speed Reference configurations are provided and compared. The design procedures were validated and compared by means of analytical equations and experimental results on a $22 \mathrm{~kW}$ test rig showing good agreement with the expected dynamics. The proposed system appears to be a good subsystem for future power distribution system targeting at more reliable transportation systems and safety critical applications.

\section{REFERENCES}

[1] F. Quentin and J. Szodruch. Aeronautics and air transport: beyond vision 2020 towards 2050 ACARE. Europe. (2010, Jun.). [Online]. Available: https://publications.europa.eu

[2] M. J. Duran and F. Barrero, "Recent advances in the design, modeling, and control of multiphase machines - Part II," IEEE Transactions on Industrial Electronics, vol. 63, DOI 10.1109/TIE.2015.2448211, no. 1, pp. 459-468, Jan. 2016.

[3] M. J. Duran and F. Barrero, "Recent advances in the design, modeling, and control of multiphase machines - Part II," IEEE Transactions on Industrial Electronics, vol. 63, DOI 10.1109/TIE.2015.2448211, no. 1, pp. 459-468, Jan. 2016.

[4] E. Levi, "Advances in converter control and innovative exploitation of additional degrees of freedom for multiphase machines," IEEE Transactions on Industrial Electronics, vol. 63, DOI 10.1109/TIE.2015.2434999, no. 1, pp. 433-448, Jan. 2016.

[5] B. Sarlioglu and C. T. Morris, "More electric aircraft: Review, challenges, and opportunities for commercial transport aircraft," IEEE Transactions on Transportation Electrification, vol. 1, DOI 10.1109/TTE.2015.2426499, no. 1, pp. 54-64, Jun. 2015.

[6] Y. Hu, Z. Q. Zhu, and M. Odavic, "Comparison of two-individual current control and vector space decomposition control for dual three-phase PMSM," IEEE Transactions on Industry Applications, vol. 53, DOI 10.1109/TIA.2017.2703682, no. 5, pp. 4483-4492, Sep. 2017.

[7] W. Allen J., W. Bruce F., and S. Gerald B., Power Generation, Operation, and Control. Wiley, 1984.

[8] U. Borup, F. Blaabjerg, and P. Enjeti, "Sharing of nonlinear load in parallel-connected three-phase converters," IEEE Trans. Ind. Appl., vol. 37, DOI 10.1109/28.968196, no. 6, pp. 1817-1823, Nov. 2001.

[9] J. M. Guerrero, J. C. Vasquez, J. Matas, L. G. de Vicuna, and M. Castilla, "Hierarchical control of droop-controlled ac and dc microgrids - a general approach toward standardization," IEEE Transactions on Industrial Electronics, vol. 58, DOI 10.1109/TIE.2010.2066534, no. 1, pp. 158172, Jan. 2011.

[10] J. Figueroa, J. Cros, and P. Viarouge, "Generalized transformations for polyphase phase-modulation motors," IEEE Transactions on Energy Conversion, vol. 21, DOI 10.1109/TEC.2005.859965, no. 2, pp. 332341, Jun. 2006.

[11] R. Bojoi, M. Lazzari, F. Profumo, and A. Tenconi, "Digital field-oriented control for dual three-phase induction motor drives," IEEE Transactions on Industry Applications, vol. 39, DOI 10.1109/TIA.2003.811790, no. 3, pp. 752-760, May. 2003. 